\title{
Health and welfare profile of Australian baby boomers who live in rented accommodation - implications for the future
}

\author{
ANNE W. TAYLOR*, RHIANNON PILKINGTON $\dagger$, \\ ELEONORA DAL GRANDE*, CONSTANCE KOURBELIS $\ddagger$ and \\ HELEN BARRY§
}

\begin{abstract}
Baby boomers who rent are often overlooked as an important sub-group. We aimed to assess the chronic conditions, risk factors, socio-economic factors and other health-related factors associated with renting in private or public housing. Data from telephone interviews conducted each month in South Australia between 2010 and 2015 were combined. Prevalence estimates were assessed for each risk factor and chronic condition by housing status. The association between housing status and variables of interest were analysed using logistic regression models adjusting for multiple covariates (age, gender, income, smoking, physical activity, area and year of data collection). Overall, 17.4 per cent of the 16,687 baby boomers interviewed were renting, either privately or using government-subsided housing. The health profile of renters (both private and public) was poorer overall, with renters more likely to have all of the chronic conditions and ten risk factors assessed. For public renters the relationships were maintained even after controlling for socio-economic and risk factor variables for all chronic diseases except osteoporosis. This research has provided empirical evidence of the considerable differences in health, socio-economic indicators and risk factors between baby boomers who rent and those who own, or are buying, their own homes.
\end{abstract}

KEY WORDS-baby boomers, Australia, risk factors, chronic disease, rented accommodation, housing.

* Population Research \& Outcome Studies, Discipline of Medicine, The University of Adelaide, Australia.

$\dagger$ School of Population Health, The University of Adelaide, Australia.

\$ School of Medicine, The University of Adelaide, Australia.

$\S$ Australian Population \& Migration Research Centre, The University of Adelaide, Australia. 


\section{Introduction}

Baby boomers (classified in Australia as born between 1946 and 1965) are frequently cited, rightly or wrongly, as the lucky generation with a perception that they are better off financially, had enhanced education opportunities and are healthier than the preceding generations (Biggs et al. 20o6; Faulkner 2007; Hamilton and Hamilton 2006; Quine and Carter 2006; Pinnegar and van den Nouwelant 2012). Baby-boomer research is often centred on demographic changes, health, pensions, superannuation and retirement plans (Humple et al. 2010; Hunter, Wang and Worsely 2007; Kubicek et al. 2010; Martin et al. 2009; Quine and Carter 2006; Taylor et al. 2014). While commentary on sea-change/tree-change moves (the move from major cities to an 'easier' life in rural/coastal communities) (Gurran and Blakely 2007; Olsberg and Winters 2005), downsizing (Wulff, Champion and Lobo 2010) and reverse mortgages (Snoke, Kendig and O'Loughlin 2011) are frequently addressed, other housing considerations, especially baby boomers who rent, are often overlooked as an important research and policy issue.

Pensions, superannuation (government-supported retirement savings based on employment income) and savings are the three funding mechanisms for retirees, although increasingly housing equity is seen as the 'fourth pillar' (Yates and Bradbury 2010). Notwithstanding recent property devaluations and housing value volatility in most developed countries, baby boomers are a cohort who have experienced a property market that has escalated considerably across their housing career and their homes are frequently a considerable and critical asset (Beer and Faulkner 2oog; Harding, King and Kelly 2002; Ong et al. 2013; Snoke, Kendig and O'Loughlin 2011). In the Australian Census, 75 per cent of baby boomers owned or were paying off their own home and 17 per cent were renting (either privately or via public housing) (Yates and Bradbury 2010). Figures for baby boomers as home-owners of 70 per cent for Europe and 8o per cent for the United States of America are comparable (Angelini, Brugiavini and Weber 2014; Laaksonen, Tarkiainen and Martikainen 2009). In Australia, public housing is for individuals or families on low to moderate incomes who are having difficulty securing housing in the private rental market. Due to the very high waiting lists and prioritisation system of offering to those with the greatest need, public housing is usually available for longterm existing tenants or for people in times of crisis, such as domestic violence sufferers, ex-prisoners or those at risk of homelessness. While some baby boomers have never owned a home, a proportion of baby boomers have also moved to rental accommodation as a result of changes in marital status (divorce, widowhood), changes in household composition 
(empty nest) or economic and health-related factors (sickness, disability, unemployment, retrenchment) (Angelini, Brugiavini and Weber 2014; Faulkner 2007; Pinnegar and van den Nouwelant 2012; Wulff, Champion and Lobo 2010). Home-ownership rates decline as age advances and equity is released as increased social and health-related support is required (such as entering aged care facilities or moving in with family members) but baby boomers have yet to reach the 'advanced' age where those forces more commonly come into play (Angelini, Brugiavini and Weber 2014; Australian Housing and Urban Research Institute (AHURI) 2004).

The advantage of owning a house into retirement is seen as insurance against rent increases, security if a future need arises and as a legacy for the next generation (Angelini, Brugiavini and Weber 2014; Faulkner 2007; Snoke, Kendig and O'Loughlin 2011). Renting, either private or public, has been shown to be related to poor health outcomes in most developed countries (Hungerford 2007; Laaksonen, Tarkiainen and Martikainen 2009). Renters more often report increased financial difficulties (Angelini, Brugiavini and Weber 2014). While baby-boomer homeowners are willing to down size to release funds for lifestyle needs (AHURI 2004; Faulkner 2007; Laaksonen, Tarkiainen and Martikainen 2009), renting baby boomers do not have that option to supplement their lifestyle choices if required (Ong et al. 2013).

Australia's current social policy context includes encouragement for ageing in place (continuing to live in the family home), extending the working years (with a non-compulsory retirement age) and increasing the age when one is eligible for the pension. Age pensions were accepted as the norm in previous retired generations (Hamilton and Hamilton 2006; Harding, King and Kelly 2002). The baby-boomer cohort, as they approach retirement, with their wide range of experiences and expectations, have relatively large differences in superannuation, savings and housing assets (Biggs et al. 20o6; Faulkner 2007; Quine and Carter 2006). Over 30 per cent of baby boomers in Australia own more than one dwelling (Beer and Faulkner 2009), while some, especially renters, are seen as being financially vulnerable and may experience economic hardships into old age (Hungerford 2007; Hunter, Wang and Worsely 2007). Vulnerability often exists with families headed by divorced women, or those who have never married or who have traditionally been low-income earners (Angelini, Brugiavini and Weber 2014; Olsberg and Winters 2005). Arguments have been made that although women will be over-represented in the renting group because of their substantially lower superannuation resources (Olsberg and Winters 2005; Snoke, Kendig and O'Loughlin 2011), low-income couple households will also be seeking assistance with their housing needs (AHURI 2008). 
Previous studies have shown that being a renter in the pre-retirement years is associated with many later-year hardships, including health and wealth (Hungerford 2007). As such, we assess the chronic conditions, risk factors, socio-economic factors and other health-related factors associated with renting, both private and public, to determine the profile of the renting group before they reach the normative retirement years. We also compare renters with home-owners.

\section{Methods}

The South Australian Monitoring and Surveillance System (SAMSS) is a telephone monitoring system designed to monitor systematically chronic disease, risk factors and other health-related issues on a regular and ongoing basis (Population Research and Outcome Studies 2004). Commencing in July 2002, a representative cross-sectional sample is randomly selected each month from all households in South Australia (SA) with a telephone connected and the number listed in the telephone directory. A letter of introduction is sent to the selected household and the person who was last to have a birthday within a 12-month period is chosen for interview.

A trained interviewer, via a computer-assisted telephone interview system, conducts the interviews. Data are collected by a contracted agency and interviews are conducted in English. Each interview takes approximately 15 minutes. At least ten call-backs are made to the telephone number selected. Replacement interviews for persons who could not be contacted or interviewed are not permitted. Interviews are conducted on a minimum of 6 oo randomly selected people (of all ages) each month. Of each interviewer's work, 10 per cent is selected at random for validation by a supervisor. The current analysis used data collected from January 2010 to December 2015. The median response rate was 63.8 per cent. No details were available for non-responders although the data were weighted to overcome potential response bias. All respondents gave informed consent to undertake the interview. Ethics approval was obtained from the ethics committee of the Department of Health and Ageing, SA (number 436/o2/ 2014).

This analysis is limited to the responses from baby boomers only $(\mathrm{N}=16,687)$. Respondents were asked if the dwelling was 'owned or being purchased', 'rented from Housing SA', 'rented privately' or 'other'. Housing SA is the government department responsible for public housing rentals. The 'other' category covered community housing and retirement village accommodation and were excluded from the analysis $(\mathrm{N}=114)$. 
Descriptive analyses were undertaken using the following demographics, chronic diseases and risk factors.

\section{Demographics}

Demographic and socio-economic variables included in the descriptive analyses consisted of age, gender, work status, highest education obtained, marital status, country of birth, household annual income and Aboriginal/Torres Strait Islanders status. The single pension in Australia is less than Aus \$20,00o per year. The lower amount was selected as the aim of the analysis was to examine vulnerable groups, especially those at the lower end of income levels. Two questions assessed the current household money situation and the family/household structure. Socio-economic status was assessed using postcodes classified into the Socio-economic Index for Areas (SEIFA) 2006 Index of Relative Socio-economic Disadvantage quintiles (Australian Bureau of Statistics (ABS) 2006). SEIFA ranks areas in Australia according to socio-economic advantage and disadvantage and is a composite score based on selected Census socio-economic variables. Questions on social capital-related factors included neighbourhood safety (yes/no), trust in the neighbourhood (yes/no), home safety (all of the time to none of the time), control over life decisions (strongly agree to strongly disagree) and transport problems (never to all of the time). Age (continuous), gender, household income and area (country SA or metropolitan Adelaide) were classified as confounders and used in the multivariate adjustments.

\section{Chronic conditions}

Respondents were asked whether a doctor had ever told them they had diabetes, cardiovascular disease (CVD), chronic obstructive pulmonary disease (COPD), arthritis or osteoporosis, or if they had asthma symptoms in the previous 12 months. In addition, respondents were asked if they had ever been diagnosed with depression, anxiety, or a stress-related or other mental health problem. A multiple chronic condition variable was created from the seven chronic conditions assessed, with two or more chronic conditions indicating multiple chronic conditions.

\section{Risk factors}

Respondents were asked whether a doctor had ever told them they have or were currently receiving treatment or medication for high blood pressure or high cholesterol. Respondents were asked to provide the time they spent 
undertaking walking, moderate or vigorous physical activity over the past week. The time was summed, with the time spent undertaking vigorous activity multiplied by a factor of two to account for its greater intensity, in order to provide an indication as to whether respondents are undertaking a sufficient level of physical activity to provide a health benefit. This is defined as $15^{\circ}$ minutes or more of activity each week (Department of Health and Ageing 1999) and has been recoded into insufficient activity (no activity, active but not sufficient) and sufficient activity.

Body mass index was derived from self-reported weight and height and recoded into three categories (underweight/normal, overweight and obese) (World Health Organization 2013). Respondents were asked how many servings of fruit and how many servings of vegetables they ate each day, with the recommendation being at least two servings of fruit and five servings of vegetables each day (National Health and Medical Research Council 2005).

Smoking status (current, ex or non) was also assessed. In addition, a multiple risk factor variable was created (physical inactivity, current smoking, inadequate consumption of fruit, inadequate consumption of vegetables, psychological distress, current high blood pressure, current high cholesterol and obesity) with a positive response to two or more risk factors indicating multiple risk factors. How respondents would rate their overall health was recoded, with fair or poor determined as low overall health status. For adjustment in the multivariate analysis only smoking status and sufficient physical activity were used, as these were the only risk factors deemed to be possible confounders.

\section{Analysis}

The weighting procedure known as 'raking' was used to weight the respondents by probability of selection within the household and by a range of socio-economic and demographic characteristics (age, gender, region, highest education level obtained, renting status, country of birth, marital status and employment status) (Dal Grande et al. 2015) using SA-specific ABS Census data so that the estimates are reflective of the structure of the population being assessed.

Data were analysed using SPSS for Windows version 24.o and Stata version 13.o. Prevalence estimates were assessed for each risk factor and chronic condition by housing status. Chi-square tests were used to compare differences in the categorical variables. The association between housing status and the chronic conditions and risk factors were analysed using logistic regression models adjusting for multiple covariates. The multivariable models controlled for demographic variables (age (continuous), income, 
area and gender) and known risk factors (smoking and physical activity) and year of data collection.

\section{Results}

The demographic profile of the three groups (home-owners or buyers, private and public renters) are detailed in Table 1. Overall 16,687 babyboomer respondents were included in the analysis (mean age $=54.1$ years, standard deviation $(\mathrm{SD})=6.04), 49.2$ per cent male with 17.4 per cent renting either privately $(10.1 \%, 95 \% \mathrm{CI}=9.7-10.6)$ or publicly $(7.3 \%$, $95 \% \mathrm{CI}=6.9-7.7)$. The mean age was 56.5 years $(\mathrm{SD}=5.9)$ for those owning or paying off their home, 55.6 years $(\mathrm{SD}=5.9)$ for private renters and 57.2 years $(\mathrm{SD}=6.2)$ for public renters. Increased proportion for public renters included those unable to work, the lower educated, those never married, those in the lowest SEIFA quintile, those earning $<$ Aus \$20,ooo per year and adults living alone.

The relationship between housing status and the social capital-related variables is highlighted in Table 2. Public renters were more likely to report not living in a safe neighbourhood, not believing people in the neighbourhood trust one another and sometimes having problems with transport.

The relationship between housing status and chronic conditions and risk factors are highlighted in Table 3. Public renters are more likely to have an increased risk of all of the chronic conditions and risk factors examined.

Using multivariable analysis after adjustment, housing status was positively associated with CVD and mental health problems for both private and public renters and for diabetes, arthritis and COPD for public renters. The odds ratios increased from owning or buying own home to privately renting to public renting for all conditions except osteoporosis. The raw and adjusted estimates are presented in Table 4 .

\section{Discussion}

This analysis has shown that there are major differences in the demographic and health profiles of baby-boomer renters and non-renters, with those renting from the government more likely to be living alone, report being unable to work as their work status, have low education levels, be in the lowest quintile of socio-economic disadvantage, have a low income and have just enough money to exist each pay period. The health profile of renters (both private and public) was poorer overall, with renters more likely to have an increased risk of having all of the chronic conditions 


\begin{tabular}{|c|c|c|c|c|c|c|c|}
\hline & \multicolumn{2}{|c|}{$\begin{array}{l}\text { Home owned or being } \\
\text { purchased }\end{array}$} & \multicolumn{2}{|c|}{ Renters (private) } & \multicolumn{2}{|c|}{ Renters (public) } & \multirow[b]{2}{*}{$p$} \\
\hline & $\mathrm{N}$ & $\%(95 \% \mathrm{CI})$ & $\mathrm{N}$ & $\%(95 \% \mathrm{CI})$ & $\mathrm{N}$ & $\%(95 \% \mathrm{CI})$ & \\
\hline \multicolumn{8}{|l|}{ Gender: } \\
\hline Male & $6,83_{1}$ & $49.6\left(4^{8.7}-5^{0.4}\right)$ & 808 & $47.8\left(45.4^{-} 5^{0.2}\right)$ & $5^{67}$ & $4^{6.5}(43 \cdot 7-49 \cdot 3)$ & 0.057 \\
\hline Female & 6,946 & $5^{0.4}\left(49 \cdot 6-5^{1.3}\right)$ & 882 & $5^{2.2}(49.8-54.6)$ & $65^{2}$ & $53.5\left(5^{0.7}-5^{6.3}\right)$ & 0.057 \\
\hline \multicolumn{8}{|l|}{ Work status: } \\
\hline Full-time employed & 6,749 & $49.0(48.2-49.8)$ & 705 & $41.7(39 \cdot 4-44 \cdot 1)$ & 189 & $15.5(13.6-17.6)$ & $<0.001$ \\
\hline Part-time employed & 3,175 & $23.0(22.3-23.8)$ & 426 & $25.2(23.2-27.4)$ & 154 & $12.6(10.9-14.6)$ & \\
\hline Unemployed & 285 & $2.1(1.8-2.3)$ & 70 & $4.2(3.3-5.2)$ & 59 & $4.8(3.8-6.2)$ & \\
\hline Engaged in home duties & $45^{8}$ & $3 \cdot 3(3.0-3.6)$ & 49 & $2.9(2.2-3.8)$ & $5^{1}$ & $4.2(3.2-5.5)$ & \\
\hline Student/other & $3^{1}$ & $0.2(0.2-0.3)$ & 21 & $1.2(0.8-1.9)$ & 7 & $0.6(0.3-1.2)$ & \\
\hline Retired & 2,187 & $15 \cdot 9\left(15 \cdot 3^{-16.5}\right)$ & 167 & $9.9\left(8.5^{-11.4}\right)$ & 220 & $18.1(16.0-20.3)$ & \\
\hline Unable to work & 870 & $6.3(5.9-6.7)$ & $25^{2}$ & $14.9\left(13.3^{-16.7}\right)$ & 537 & $44.0\left(4^{1.3}-4^{6.8}\right)$ & \\
\hline \multicolumn{8}{|l|}{ Highest education level: } \\
\hline No schooling to secondary & 6,628 & $48.1(47 \cdot 3-49.0)$ & 911 & $54.0\left(5^{1.6-5^{6.3}}\right)$ & 919 & $75.5(73.1-77.9)$ & $<0.001$ \\
\hline Trade, certificate, diploma & 4,662 & $33.9(33.1-34.6)$ & 542 & $32.1(29.9-34.4)$ & 255 & $21.0(18.8-23.3)$ & \\
\hline Degree or higher ${ }^{1}$ & 2,481 & $18.0(17.4-18.7)$ & 235 & $13.9\left(12.3^{-1} 5.6\right)$ & 43 & $3.5(2.6-4 \cdot 7)$ & \\
\hline \multicolumn{8}{|l|}{ Marital status: } \\
\hline Married/living with partner & 10,005 & $72.6(71.9-73.4)$ & 630 & $37 \cdot 3(35 \cdot 0-39 \cdot 6)$ & 276 & $22.6\left(20.3^{-25} .0\right)$ & $<0.001$ \\
\hline Separated/divorced & 1,914 & $13.9\left(13.3^{-14.5}\right)$ & 686 & $40.6\left(3^{8.3-42.9)}\right.$ & $5^{20}$ & $42.6(39 \cdot 9-45 \cdot 4)$ & \\
\hline Widowed & $5^{63}$ & $4.1(3.8-4.4)$ & 54 & $3.2(2.4-4.1)$ & 94 & $7.7(6.4-9.4)$ & \\
\hline Never married & 1,285 & $9 \cdot 3(8.8-9.8)$ & $3^{17}$ & $18.8(17.0-20.7)$ & 323 & $26.5(24.1-29.0)$ & \\
\hline \multicolumn{8}{|l|}{ SEIFA: } \\
\hline Lowest quintile & 2,335 & $17.0\left(16.4^{-17.6)}\right.$ & 402 & $23.8(21.8-25.9)$ & $5^{20}$ & $4^{2.7}(39 \cdot 9-45 \cdot 5)$ & $<0.001$ \\
\hline Low quintile & 2,606 & $18.9(18.3-19.6)$ & 347 & $20.5(18.7-22.5)$ & 300 & $24.6\left(22.3^{-27.1)}\right.$ & \\
\hline Middle quintile & 2,682 & $19.5(18.8-20.2)$ & 309 & $18.3\left(16.5^{-20.2)}\right.$ & 221 & $18.1(16.1-20.4)$ & \\
\hline High quintile & 2,859 & $20.8(20.1-21.5)$ & 383 & $22.7(20.7-24.7)$ & 104 & $8.5(7.1-10.2)$ & \\
\hline Highest quintile & 3,277 & $23.8(23.1-24.5)$ & 249 & $14.7(13.1-16.5)$ & 73 & $6.0(4.8-7.5)$ & \\
\hline \multicolumn{8}{|l|}{ Country of birth: } \\
\hline Australia & 9,782 & $71.0(70.2-71.8)$ & 1,091 & $64.6(62.3-66.8)$ & 907 & $74 \cdot 4(71.9-76.8)$ & $<0.001$ \\
\hline United Kingdom/Ireland & 1,862 & $13.5(13.0-14.1)$ & 262 & $15.5\left(13.9^{-17.3)}\right.$ & 183 & $15.0(13.1-17.1)$ & \\
\hline
\end{tabular}




\begin{tabular}{|c|c|c|c|c|c|c|c|}
\hline Other & 2,115 & $15 \cdot 3(14.8-16.0)$ & 328 & $19.4(17.6-21.4)$ & 125 & $10.2(8.6-12.0)$ & \\
\hline \multicolumn{8}{|l|}{ ATSI: } \\
\hline No & 13,696 & $99 \cdot 4(99 \cdot 3-99 \cdot 5)$ & 1,664 & $98.5(97.8-99.0)$ & $1,14^{6}$ & $94.0\left(92.5^{-95.2}\right)$ & \multirow[t]{2}{*}{$<0.001$} \\
\hline Yes & 69 & $0.5(0.4-0.6)$ & 16 & $1.0(0.6-1.6)$ & 64 & $5.2(4.1-6.6)$ & \\
\hline \multicolumn{8}{|l|}{ Household income (Aus \$): } \\
\hline 20,001 or more & 10,364 & $75.2\left(74.5^{-75.9}\right)$ & 1,137 & $67 \cdot 3\left(6_{5} .0-69.5\right)$ & 394 & $32.3(29.8-35.0)$ & \multirow[t]{3}{*}{$<0.001$} \\
\hline$<20,001$ & 787 & $5 \cdot 7(5 \cdot 3-6.1)$ & $25^{2}$ & $14.9\left(13.3^{-16.7)}\right.$ & 545 & $44 \cdot 7(41.9-47 \cdot 5)$ & \\
\hline Not stated & 2,627 & $19.1(18.4-19.7)$ & 301 & $17.8(16.1-19.7)$ & 280 & $23.0(20.7-25.4)$ & \\
\hline \multicolumn{8}{|l|}{ Money situation: } \\
\hline Spending more money than get & $4^{69}$ & $3 \cdot 4(3.1-3 \cdot 7)$ & 104 & $6.1(5.1-7 \cdot 4)$ & 70 & $5 \cdot 7\left(4 \cdot 5^{-7.2}\right)$ & \multirow[t]{6}{*}{$<0.001$} \\
\hline $\begin{array}{l}\text { Have just enough money to get through to } \\
\text { the next pay day }\end{array}$ & 2,387 & $17 \cdot 3(16.7-18.0)$ & $5^{82}$ & $34.4\left(3^{2.2-}-3^{6.7}\right)$ & $5^{8} 3$ & $47.8\left(45^{.0}-5^{0.6}\right)$ & \\
\hline $\begin{array}{l}\text { There's some money left over each week } \\
\text { but just spend it }\end{array}$ & 532 & $3.9(3.6-4.2)$ & 71 & $4 \cdot 2(3 \cdot 4-5 \cdot 3)$ & 55 & $4.5\left(3 \cdot 5^{-5} \cdot 8\right)$ & \\
\hline Can save a bit every now and then & 7,268 & $5^{2.7}\left(5^{1.9-53.6)}\right.$ & 728 & $43.1(40.7-45 \cdot 5)$ & 444 & $3^{6.4}(33.8-39.1)$ & \\
\hline Can save a lot & 2,705 & $19.6(19.0-20.3)$ & $16_{5}$ & $9.7\left(8.4^{-1} 1.2\right)$ & $3^{8}$ & $3.1(2.3-4.2)$ & \\
\hline Don’t know/refused & 417 & $3.0(2.8-3.3)$ & 40 & $2.4(1.7-3.2)$ & $3^{0}$ & $2.4(1.7-3.4)$ & \\
\hline \multicolumn{8}{|l|}{ Family structure: } \\
\hline $\begin{array}{l}\text { A family with a child or children living with } \\
\text { both biological or adoptive parents }\end{array}$ & 3,111 & $22.6\left(21.9^{-2} 3 \cdot 3\right)$ & $15^{2}$ & $9.0(7.7-10.4)$ & $4^{6}$ & $3.8(2.9-5.0)$ & \multirow[t]{11}{*}{$<0.001$} \\
\hline A step or blended family & 178 & $1.3(1.1-1.5)$ & $4^{1}$ & $2.4(1.8-3.2)$ & 13 & $1.1(0.6-1.9)$ & \\
\hline A sole-parent family & 420 & $3.0(2.8-3.3)$ & 132 & $7.8(6.6-9.2)$ & 75 & $6.2(4.9-7.6)$ & \\
\hline Shared care parenting & 13 & $0.1(0.1-0.2)$ & 17 & $1.0(0.6-1.6)$ & 4 & 0.3 & \\
\hline Adult living alone & 2,371 & $17.2(16.6-17.8)$ & 684 & $40.5\left(3^{8.2-42.8)}\right.$ & 710 & $5^{8.2}\left(55 \cdot 4^{-61.0}\right)$ & \\
\hline Adult living with partner and no children & 6,021 & $43 \cdot 7(42.9-44 \cdot 5)$ & 405 & $24.0(22.0-26.1)$ & 204 & $16.7(14.7-18.9)$ & \\
\hline Related adults living together & 1,140 & $8.3(7.8-8.7)$ & 120 & $7.1(5.9-8.4)$ & 126 & $10.4(8.8-12.2)$ & \\
\hline Unrelated adults living together & 167 & $1.2(1.0-1.4)$ & 95 & $5.6(4.6-6.8)$ & 22 & $1.8(1.2-2.8)$ & \\
\hline $\begin{array}{l}\text { Related adults/children living together } \\
\text { (including step families) }\end{array}$ & 284 & $2.1(1.8-2.3)$ & 35 & $2.1\left(1.5^{-2.8)}\right.$ & 12 & $1.0(0.6-1.7)$ & \\
\hline Other/refused & 73 & $0.5(0.4-0.7)$ & 10 & $0.6\left(0.3^{-1.1}\right)$ & 7 & $0.5\left(0.3^{-1.1}\right)$ & \\
\hline Overall & 13,778 & 100.0 & 1,690 & 100.0 & 1,219 & 100.0 & \\
\hline
\end{tabular}

Notes: The weighting of the data can result in rounding discrepancies or totals not adding. Refused were not reported. CI: confidence interval. SEIFA: Socio-economic Index for Areas. ATSI: Aboriginal/Torres Strait Islanders. 1. University education. 


\begin{tabular}{|c|c|c|c|c|c|c|c|}
\hline & \multicolumn{2}{|c|}{$\begin{array}{l}\text { Home owned or being } \\
\text { purchased }\end{array}$} & \multicolumn{2}{|c|}{ Renters (private) } & \multicolumn{2}{|c|}{ Renters (public) } & \multirow[b]{2}{*}{$p$} \\
\hline & $\mathrm{N}$ & $\%(95 \% \mathrm{CI})$ & $\mathrm{N}$ & $\%(95 \% \mathrm{CI})$ & $\mathrm{N}$ & $\%(95 \% \mathrm{CI})$ & \\
\hline \multicolumn{8}{|c|}{ Neighbourhood is a safe place: } \\
\hline Yes & 12,930 & $93.8(93.4-94.2)$ & 1,495 & $88.5(86.8-89.9)$ & $93^{2}$ & $76.5(74.0-78.8)$ & \multirow[t]{3}{*}{$<0.001$} \\
\hline No & 468 & $3.4(3.1-3 \cdot 7)$ & 123 & $7 \cdot 3(6.1-8.6)$ & 197 & $16.1(14.2-18.3)$ & \\
\hline Don't know/not sure & $3^{80}$ & $2.8\left(2.5^{-3.0}\right)$ & 72 & $4 \cdot 3(3 \cdot 4-5 \cdot 3)$ & 90 & $7 \cdot 4(6.0-9.0)$ & \\
\hline \multicolumn{8}{|c|}{ Neighbourhood people generally trust one another: } \\
\hline Yes & $11,35^{2}$ & $82.4(81.7-83.0)$ & 1,207 & $71.4(69.2-73.5)$ & $6_{5}^{6}$ & $53.8\left(5^{1.0}-5^{6.6}\right)$ & \multirow[t]{3}{*}{$<0.001$} \\
\hline No & 698 & $5.1(4 \cdot 7-5 \cdot 4)$ & $14^{6}$ & $8.6\left(7.4^{-10.1)}\right.$ & 305 & $25.0(22.7-27.5)$ & \\
\hline Don't know/not sure & 1,728 & $12.5(12.0-13.1)$ & 337 & $20.0(18.1-21.9)$ & $25^{8}$ & $21.2(19.0-23.5)$ & \\
\hline \multicolumn{8}{|l|}{ Feel safe in home: } \\
\hline All of the time & 10,463 & $75.9(75.2-76.6)$ & 1,192 & $70.5(68.3-72.6)$ & 703 & $57.7(54.9-60.4)$ & \multirow[t]{4}{*}{$<0.001$} \\
\hline Most of the time & 3,112 & $22.6(21.9-23.3)$ & 444 & $26.3(24.2-28.4)$ & 419 & $34 \cdot 3(31.7-37.0)$ & \\
\hline Some of the time & 161 & $1.2(1.0-1.4)$ & $4^{2}$ & $2.5(1.8-3.3)$ & 79 & $6.5(5 \cdot 3-8.0)$ & \\
\hline None of the time & 33 & $0.2(0.2-0.3)$ & 9 & $0.5\left(0.3^{-1.0}\right)$ & 14 & $1.2(0.7-1.9)$ & \\
\hline \multicolumn{8}{|c|}{ Control over the decisions that affect my life: } \\
\hline Strongly agree & 6,722 & $48.8(48.0-49.6)$ & $7^{6} 5$ & $45.2(42.9-47.6)$ & 453 & $37.2\left(34.5^{-39.9)}\right.$ & \multirow[t]{5}{*}{$<0.001$} \\
\hline Agree & 6,171 & $44.8(44.0-45.6)$ & 755 & $44 \cdot 7(42.3-47.1)$ & 622 & $5^{1.0}\left(4^{8.2-53.8)}\right.$ & \\
\hline Neutral/don't know & 235 & $1.7\left(1.5^{-1.9)}\right.$ & 45 & $2.7(2.0-3.5)$ & 55 & $4.5\left(3.5^{-5} \cdot 8\right)$ & \\
\hline Disagree & $5^{65}$ & $4.1(3.8-4.4)$ & 109 & $6.5(5 \cdot 4-7 \cdot 7)$ & 73 & $6.0(4.8-7.4)$ & \\
\hline Strongly disagree & 83 & $0.6\left(0.5^{-0.8}\right)$ & 16 & $0.9(0.6-1.5)$ & 17 & $1.4\left(0.9^{-2.2}\right)$ & \\
\hline \multicolumn{8}{|l|}{ Problems with transport: } \\
\hline Never & 12,899 & $93.6(93.2-94.0)$ & $1,5^{\circ 0}$ & $88.8(87.2-90.2)$ & $93^{8}$ & $76.9\left(74.5^{-79.2)}\right.$ & \multirow[t]{4}{*}{$<0.001$} \\
\hline Sometimes & $75^{2}$ & $5.5(5.1-5.8)$ & 161 & $9.5(8.2-11.0)$ & 192 & $15.7(13.8-17.9)$ & \\
\hline All the time & 125 & $0.9(0.8-1.1)$ & 29 & $1.7(1.2-2.5)$ & $8_{5}$ & $6.9(5.6-8.5)$ & \\
\hline Overall & 13,778 & 100.0 & 1,690 & 100.0 & 1,219 & 100.0 & \\
\hline
\end{tabular}


T А в L E 3. Chronic condition and risk factor profile of baby boomers by dwelling type, January 20 Io to December 2015

\begin{tabular}{|c|c|c|c|c|c|c|c|}
\hline & \multicolumn{2}{|c|}{$\begin{array}{l}\text { Home owned or being } \\
\text { purchased }\end{array}$} & \multicolumn{2}{|c|}{ Renters (private) } & \multicolumn{2}{|c|}{ Renters (public) } & \multirow[b]{2}{*}{$p$} \\
\hline & $\mathrm{N}$ & $\%(95 \% \mathrm{CI})$ & $\mathrm{N}$ & $\%(95 \% \mathrm{CI})$ & $\mathrm{N}$ & $\%(95 \% \mathrm{CI})$ & \\
\hline Diabetes & 1,296 & $9.4(8.9-9.9)$ & 195 & $11.6(10.1-13.2)$ & 257 & $21.0(18.8-23.4)$ & $<0.001$ \\
\hline Asthma & 1,566 & $11.4(10.8-11.9)$ & $25^{\circ}$ & $14.8(13.2-16.6)$ & 262 & $21.5(19.3-23.9)$ & $<0.001$ \\
\hline CVD & 953 & $6.9\left(6.5^{-7.4)}\right.$ & 178 & $10.5(9.2-12.1)$ & 218 & $17.9(15.8-20.1)$ & $<0.001$ \\
\hline COPD & 640 & $4.6(4.3-5.0)$ & 128 & $7.6(6.4-9.0)$ & 172 & $14.1\left(12.3^{-16.2}\right)$ & $<0.001$ \\
\hline Arthritis & 3,937 & $28.6(27.8-29.3)$ & $5^{27}$ & $31.2(29.0-33.4)$ & $55^{2}$ & $45 \cdot 3\left(42.5-4^{8.1}\right)$ & $<0.001$ \\
\hline Osteoporosis & 675 & $4.9\left(4.5^{-5} 5 \cdot 3\right)$ & 90 & $5 \cdot 3\left(4.3^{-6.5}\right)$ & 118 & $9.7(8.1-11.4)$ & $<0.001$ \\
\hline Current mental health problem & 2,374 & $17.2(16.6-17.9)$ & 430 & $25 \cdot 4(23.4-27.6)$ & $5^{00}$ & $41.0(38.3-43.8)$ & $<0.001$ \\
\hline At least one chronic condition & $7,14^{8}$ & $5^{1.9}\left(5^{1.0-5^{2.7}}\right)$ & 983 & $5^{8.2}\left(55^{8} 8-60.5\right)$ & 929 & $76.2(73 \cdot 7-78.5)$ & $<0.001$ \\
\hline High blood pressure & 3,681 & $26.7(26.0-27.5)$ & 463 & $27.4(25 \cdot 3-29.6)$ & 441 & $3^{6.2}(33.5-38.9)$ & $<0.001$ \\
\hline High cholesterol & 3,232 & $23.5(22.8-24.2)$ & 401 & $23.7(21.8-25.8)$ & 400 & $32.8(30.3-35.5)$ & $<0.001$ \\
\hline Insufficient physical activity & 7,169 & $53.1\left(5^{2.2-53.9)}\right.$ & 966 & $5^{8.7}\left(5^{6.3-61.0)}\right.$ & 806 & $67.8(65.1-70.4)$ & $<0.001$ \\
\hline Obese & 3,804 & $28.8(28.1-29.6)$ & 576 & $36.2(33.9-38.6)$ & 487 & $43.9(41.0-46.8)$ & $<0.001$ \\
\hline Current smoker & $2,04^{2}$ & $14.8(14.2-15.4)$ & $5^{18}$ & $30.7(28.5-32.9)$ & $45^{1}$ & $37.0(34.3-39.8)$ & $<0.001$ \\
\hline Less than five servings of vegetables per day & 12,016 & $87.6(87.1-88.2)$ & 1,534 & $91.2(89.7-92.4)$ & $1,08_{3}$ & $90.3(88.5-91.8)$ & $<0.001$ \\
\hline Less than two servings of fruit per day & 7,579 & $55 \cdot 1(54 \cdot 3-55 \cdot 9)$ & 1,048 & $62.0(59 \cdot 7-64 \cdot 3)$ & 828 & $68.3(65.6-70.8)$ & $<0.001$ \\
\hline Fair or poor self-reported health & 2,390 & $17.3(16.7-18.0)$ & $44^{8}$ & $26.5\left(24.5^{-28} .7\right)$ & 572 & $4^{6.9}(44 \cdot 1-49 \cdot 7)$ & $<0.001$ \\
\hline At least one health risk factor & $8,5^{18}$ & $67.8(66.9-68.6)$ & 1,171 & $77.8(75 \cdot 7-79.8)$ & 898 & $86.2(84.0-88.2)$ & $<0.001$ \\
\hline
\end{tabular}

Notes: The weighting of the data can result in rounding discrepancies or totals not adding. Don't know/refused were not reported. Multiple risk factors included high blood pressure, high cholesterol, insufficient physical activity, obesity, current smoking and eating less than two servings of fruit or five servings of vegetables per day. CI: confidence interval. CVD: cardiovascular disease. COPD: chronic obstructive pulmonary disease. 
T А В L 4 . Odds ratio (95\% confidence interval) for chronic diseases according to housing status among baby boomers

\begin{tabular}{|c|c|c|c|c|}
\hline & \multicolumn{2}{|l|}{ Private renting } & \multicolumn{2}{|l|}{ Public renting } \\
\hline & Unadjusted & Multivariable adjusted & Unadjusted & Multivariable adjusted \\
\hline Diabetes & $1.26\left(0.99^{-1.59)}\right.$ & $1.25(0.98-1.59)$ & $2.57(2.07-3.18) * *$ & $2.03(1.59-2.59) * *$ \\
\hline Asthma & $1.35(1.08-1.69)^{* *}$ & $1.22(0.96-1.54)$ & $2.14(1.73-2.63) * *$ & $1.78(1.41-2.24)^{* *}$ \\
\hline CVD & $1.59\left(1.23^{-2.04}\right)^{* *}$ & $1.53(1.17-2.00) * *$ & $2.93\left(2.3^{6-3.63}\right)^{* *}$ & $1.97\left(1.53^{-2.53}\right)^{* *}$ \\
\hline COPD & $1.69\left(1.23^{-2.31}\right)^{* *}$ & $1.30(0.92-1.82)$ & $3.37(2.62-4.32) * *$ & $2.07\left(1.54^{-2.79}\right) * *$ \\
\hline Arthritis & $1.13(0.96-1.34)$ & $1.07(0.90-1.28)$ & $2.07\left(1.74^{-2.45}\right)^{* *}$ & $1.43(1.18-1.73)^{* *}$ \\
\hline Osteoporosis & $1.09\left(0.78-1.5^{2}\right)$ & $0.97(0.69-1.37)$ & $2.08(1.60-2.69) * *$ & $1.18(0.88-1.59)$ \\
\hline Mental health problem & $1.64\left(1.3^{6-1.97}\right)^{* *}$ & $1.30\left(1.07-1.5^{8}\right) * *$ & $3.34(2.8 \mathrm{o}-3.98) * *$ & $1.79\left(1.4^{6-2.20}\right)^{* *}$ \\
\hline At least one NCD & $1.27(1.08-1.50)^{* *}$ & $1.13\left(0.95^{-1.34}\right)$ & $3.18(2.57-3.93) * *$ & $2.00\left(1.59^{-2.51}\right)^{* *}$ \\
\hline
\end{tabular}

Notes: Multivariable models adjusted for age, gender, smoking status, income, sufficient physical activity, area, year of survey. Reference category = home owned or being purchased. COPD: chronic obstructive pulmonary disease. CVD: cardiovascular disease. NCD: non-communicable disease.

Significance level: $* * p<0.01$. 
(except osteoporosis) and risk factors assessed. In most instances there was a trend from home-owners to private renters to public renters with, in all instances, the public renters having the worst health outcomes. For those publicly renting these relationships were maintained even after controlling for socio-economic and risk factor variables, while for those privately renting the relationships for CVD and mental health problems were maintained after adjustment.

The overall prevalence of baby-boomer renters $(17.4 \%)$ is in line with ABS published figures, adding reliability to these results (ABS 2011). Of concern is the large number of dwellings this represents. As argued by Yates and Bradbury (2010), public housing is decreasing in number and scale and while private rental might have more attributes, affordability will be a major issue (AHURI 2008). While market forces determine renting costs, baby boomers will be indebted to this private market with little or no fall back, with the Australian Commonwealth Rent Assistance programme limited and not covering the gap when the more expensive private rental housing market is assessed (Cartwright 2007). The demand for government-funded housing will increase, as will the need to diversify housing for this heterogeneous group. One questions whether the suitability, number, location, amenities, quality and variety of public housing in Australia will be sufficient to cover the requirements of this population into the future. These issues are compounded by current government policy aimed at keeping older people in their home rather than in a nursing home or similar accommodation offering assistance. Research has indicated that Australian baby boomers will want to live independently and within the community rather than residential aged care or retirement villages (Quine and Carter 2006). They also do not want to be institutionalised or live with their children (Olsberg and Winters 2005; Quine and Carter 2006).

In common with other studies was the large proportion of one-person households who are renting (40.5 and $58.2 \%$ for private and government renters, respectively). As argued by Demey et al. (2013), this group regularly lack social, economic and family resources; this is often particularly true for men in this situation. Notwithstanding, single women, frequently affected by inadequate superannuation funds, are also vulnerable (Snoke, Kendig and O'Loughlin 2011). Research has shown that relationship breakdown is one of the most important reasons for renting status later in life and although women who are living alone are often satisfied with the arrangement, this is limited to those owning their home whereas women baby boomers who are renters are fearful for their future (Beer and Faulkner 2009; Olsberg and Winters 2005). Other major differences were also found between renters and home-owners in terms of family structure. The differences 


\section{Anne W. Taylor et al.}

have implications for many other issues, including care-giving status, social isolation, availability of informal care and shared financial resources, indicating a need for further research in this area.

While health is a major concern for pre-retirement baby boomers, it is usually centred on maintaining optimal health for as long as possible (Hamilton and Hamilton 2006; Humple et al. 2010; Hunter, Wang and Worsely 2007). As this research has shown, the health of baby-boomer renters is already compromised. Comparisons with the latest Australian Census $(2011 / 12)$ data indicate that the overall prevalence estimates obtained in this analysis for baby boomers are similar to Australian and SA estimates. What is of concern is the high prevalence estimates for all of the variables assessed for both of the renting groups. While babyboomer renters may have future financial concerns regarding housing options, the concerns regarding decline in health should already have manifested. As argued by Hamilton and Hamilton (2006), lower-income retirees, including renters, will not be looking forward to a 'golden age of freedom and relaxation' as will the more affluent baby boomers.

Overall, self-reported health also shows marked variation by housing status, with nearly $5^{\circ}$ per cent of government renters reporting fair or poor self-reported health. Previous research has found this question to be a strong indicator of future health-care use and mortality (Gill et al. 2009). Seen as a perceived health status measure, studies have shown relationships between social gradients of health and health equity although others have argued that self-reporting poor health is a justification for early retirement from the workforce or as an excuse for low income (Hungerford 2007). Our cross-sectional study shows both high levels of non-employment and low income for the renting group but the study is not able to determine what came first - the poor health or the renting status.

The five social capital-related questions assessed in this research were not meant to cover the broad gamut of social capital but rather serve as indications of community and social life. The fact that a trend from home-ownership to private renter to public renters existed for the variables examined is reinforced by previous research that shows often renters are less connected to their neighbourhood and this can indicate some level of social exclusion (Hungerford 2007). Coupled with this is the fact that budget rental accommodation is often not in sought-after areas, with the result that services can be compromised. Previous Australian research has shown dissatisfaction with safety and overall neighbourhoods for all renters (Parkinson et al. 2014), while our research has particularly indicated the dissatisfaction of the public housing renters. 
Weaknesses of the study include the inability to determine the length of the renting experience, and the lack of measures of wealth and other financial determinants as opposed to income (Snoke, Kendig and O'Loughlin 2011). While renting can be an indication of hardship, it does not provide information on the social and economic environment in which the person lives or the level of wealth. In addition, the cross-sectional nature of the data collection and the fact that the implications of cause and effect cannot be examined, is a weakness. This is important in this study, as we have no knowledge of whether the move to rental accommodation occurred so as to release funds for medical, health and personal care expenses. Beer and Faulker (2009) report that up to 85 per cent of private renters have previously been a home-owner or were paying off a home prior to their renting; this could not be assessed in this study. A further weakness of the study is the inability to determine whether the respondent was living rent-free or was paying rent to a friend or family who owned the dwelling. This would imply that the number renting privately may be an under-estimation. A strength of this study is being able to distinguish between private and public renters. While public renters are often seen as disadvantaged (Laaksonen, Tarkiainen and Martikainen 2009), we were able to show that the associations were apparent even after adjustment by socio-economic factors and, in most cases, the private rental group were also experiencing poor health outcomes.

Affordable, accessible and appropriate housing contributes to health and wellbeing (Demey et al. 2013). This research has provided empirical evidence of the considerable differences in health, socio-economic indicators and risk factors between baby boomers who rent and those who own, or are buying, their own homes. This analysis has added to the notion, as argued by others, that home-ownership status should be regarded as important an indicator as other commonly cited indicators, such as education level and income, when assessing health (Laaksonen, Tarkiainen and Martikainen 2009). The results of this research will fill important gaps for this subgroup of baby boomers who are going to put increased pressure on formal government support networks including health, housing and welfare (Martin et al. 2009). This may be in conflict with the current government policy of increasing individual responsibility for old-age needs and support (Hamilton and Hamilton 2006; Quine and Carter 2006).

\section{Acknowledgements}

SAMSS is owned by the Department of Health and Ageing, SA, Australia. All collected source data are maintained and managed by Population Research \& 
Outcome Studies, University of Adelaide. The opinions expressed in this work are those of the authors and may not represent the position or policy of the Department for Health and Ageing.

\section{References}

Angelini, V., Brugiavini, A. and Weber, G. 2014. The dynamics of homeownership among the $5^{\mathrm{O}+}$ in Europe. Journal of Population and Economics, 27, 3, 797-823.

Australian Bureau of Statistics (ABS) 2006. Socio-economic Indexes for Areas, Australia. Available online at http://www.abs.gov.au/websitedbs/censushome.nsf/home/ seifa [Accessed 1 October 2014].

Australian Bureau of Statistics (ABS) 2011 . ABS Information Paper: Census of Population and Housing-Products and Services: TableBuilder Pro. Available online at http:// www.abs.gov.au/ausstats/abs@.nsf/lookup/201 1.0.55.001 Main\%2oFeatures 16 2011 [Accessed 1 October 2014].

Australian Housing and Urban Research Institute (AHURI) 2004. Housing Futures in an Ageing Australia. Issue 43. Available online at https://www.ahuri.edu.au/ research/research-and-policy-bulletins/43 [Accessed 1 October 2014].

Australian Housing and Urban Research Institute (AHURI) 2008. Rental Housing for Lower-income Older Australians. Issue 96. Available online at https://www.ahuri.edu. $\mathrm{au} /$ data/assets/pdf_file/oo17/3o86/AHURI_RAP_Issue_96_Rental_housing_ provision_for_lower_income_older_Australians.pdf [Accessed 1 October 2014].

Beer, A. and Faulkner, D. 20o9. 2 Ist Century Housing Careers and Australia's Housing Future. Final Report 128, Australian Housing and Urban Research Institute. Available online at http://www.ahuri.edu.au/research/final-reports/1 28 [Accessed 1 October 2014].

Biggs, S., Phillipson, C., Money, A. M. and Leach, R. 2006. The age-shift: observations on social policy, ageism and the dynamics of the adult lifecourse. Journal of Social Work Practice, 20, 3, 239-50.

Cartwright, C. M. 2007. Affordable rental housing for older people in Australia. What do older people want? Annals of the New York Academy of Sciences, 11 14, 1, $25^{8-66 .}$

Dal Grande, E., Chittleborough, C. R., Campostrini, S. and Taylor, A. W. 2015. The effect of survey raked weighting techniques on health estimates in an Australian population health surveillance system. American Journal of Epidemiology, 182, 6, $544^{-} 5^{6 .}$

Demey, D., Berrington, A., Evandrou, M. and Falkingham, J. 2013. Pathways into living alone in mid-life: diversity and policy implications. Advances in Life Course Research, 18, 3, 161-74.

Department of Health and Ageing 1999. BeActive Australia National Physical Activity Guidelines for Adults. Available online at http://www.health.gov.au/internet/ main/publishing.nsf/Content/health-pubhlth-strateg-phys-act-guidelines\# apaadult [Accessed 1 October 2014].

Faulkner, D. 2007. The older population and changing housing careers: implications for housing provision. Australasian Journal on Ageing, 26, 4, 152-6.

Gill, T., Taylor, A. W., Broderick, D., Avery, J. and Dal Grande, E. 20o9. Self-reported overall health status: implications for intervention strategies. Australasian Medical Journal, 1, 8, 44-57. 
Gurran, N. and Blakely, E. 2007. Suffer a sea change? Contrasting perspectives towards urban policy and migration in coastal Australia. Australian Geographer, $3^{8,1,113-31 . ~}$

Hamilton, M. and Hamilton, C. 2006. Baby Boomers and Retirement. Dreams, Fears and Anxieties. Discussion Paper 89, The Australian Institute. Available online at http:// tai.org.au/documents/dp_fulltext/DP89.pdf [Accessed 1 October 2014 ].

Harding, A., King, A. and Kelly, S. 2002. Trends in the Incomes and Assets of Older Australians. Discussion Paper 58, National Centre for Social and Economic Modelling. Available online at http://www.natsem.canberra.edu.au/storage/ cp2001_o10.pdf [Accessed 1 October 2014].

Humple, N., O'Loughlin, K., Wells, Y. and Kendig, H. 2010. The health of Australian baby boomers. Australasian Journal of Ageing, 29, 1, 8-13.

Hungerford, T. L. 2007. The persistence of hardship over the life course. Research on Aging, 29, 6, 491 .

Hunter, W., Wang, W. and Worsely, A. 2007. Retirement planning and expectations of Australia's Babyboomers. Are they ready to retire? Annals of the New York Academy of Sciences, $1114,267-78$.

Kubicek, B., Korunka, C., Hoonakker, P. and Raymo, J. M. 2010. Work and family characteristics as predictors of early retirement in married men and women. Research on Aging, 32, 4, 467-98.

Laaksonen, M., Tarkiainen, L. and Martikainen, P. 2009. Housing wealth and mortality: a register linkage study of the Finnish population. Social Science and Medicine, $69,5,754^{-60 .}$

Martin, L. G., Freedman, V. A., Schoeni, R. F. and Andreski, P. M. 20o9. Health and functioning among baby boomers approaching 6o. Journal of Gerontology, $\mathbf{6 4} \mathbf{B}, 3$, $369-77$.

National Health and Medical Research Council 2005. The Dietary Guidelines for Australians. Available online at www.nhmrc.gov.au/_files_nhmrc/publication/ attachments/n31.pdf [Accessed 22 November 2011].

Olsberg, D. and Winters, M. 2005. Ageing in Place: Intergenerational and Intra-familial Housing Transfers and Shifts in Later Life. AHURI Final Report 88, Australian Housing and Urban Research Institute. Available online at http://www.ahuri. edu.au/research/final-reports/88 [Accessed 1 October 2014].

Ong, R., Haffner, M., Wood, G., Jefferson, T. and Austen, S. 2013. Assets, Debt and the Drawdown of Housing Equity by an Ageing Population. AHURI Positioning Paper 153 , Australian Housing and Urban Research Institute. Available online at http://melbourneinstitute.unimelb.edu.au/assets/documents/hilda-bibliography/other-publications/2013/Ong_etal_Assets_debt_and_the_drawdown_of_housing_equity_by_ an_ageing_population.pdf [Accessed 1 October 2014].

Parkinson, S., Ong, R., Cigdem, M. and Taylor, E. 2014. Wellbeing Outcomes of Lower Income Renters: A Multilevel Analysis of Area Effects. AHURI Final Report 226, Australian Housing and Urban Research Institute. Available online at https:// www.ahuri.edu.au/_data/assets/pdf_file/ooo8/2123/AHURI_Final_Report_ No226_Wellbeing-outcomes-of-lower-income-renters-a-multilevel-analysis-of-areaeffects.pdf [Accessed 1 October 2014].

Pinnegar, S. and van den Nouwelant, R. 201 2. Understanding Housing and Location Choices of Retiring Australians in the 'Baby Boom' Generation. Scoping report prepared for the National Housing Supply Council, City Futures Research Centre. Available online at http://www.treasury.gov.au/PublicationsAndMedia/ Publications/2012/housing-in-the-baby-boom-generation [Accessed 1 October $2014]$. 


\section{Anne W. Taylor et al.}

Population Research and Outcome Studies 2004. South Australian Monitoring and Surveillance System (SAMSS) Technical Report. Report I/o4: Survey Methodology. South Australia Department of Health. Available online at https://health.adelaide.edu.au/pros/docs/reports/report_samss_tech_paper.pdf [Accessed 1 October 2014].

Quine, S. and Carter, S. 2006. Australian baby boomers' expectations and plans for their old age. Australasian Journal of Ageing, 25, 1, 3-8.

Snoke, M., Kendig, H. and O'Loughlin, K. 2011. Australian evidence of baby boomers' financial security: a review. Journal of Economic and Social Policy, 14, 1, Article 8.

Taylor, A. W., Pilkington, R., Feist, H., Dal Grande, E. and Hugo, G. 2014. A survey of retirement intentions of baby boomers: an overview of health, social and economic determinants. BMC Public Health, 14, 355.

World Health Organization 2013. BMI Classification. Available online at http://apps. who.int/bmi/index.jsp?introPage=intro_3.html [Accessed 29 April 2013].

Wulff, M., Champion, A. and Lobo, M. 2010. Household diversity and migration in mid-life: understanding residential mobility among $45^{-6} 4$ year olds in Melbourne, Australia. Population, Space and Place, 16, 4, 307-21.

Yates, J. and Bradbury, B. 2010. Home ownership as a (crumbling) fourth pillar of social insurance in Australia. Journal of Housing and the Built Environment, 25, 193-211.

Accepted 6 September 2017 ; first published online 26 October 2017

Address for correspondence:

Anne W. Taylor,

Population Research \& Outcome Studies, Discipline of Medicine,

L7, SAHMRI, North Terrace, The University of Adelaide,

Adelaide,

South Australia, Australia

E-mail: Anne.taylor@adelaide.edu.au 